\title{
HPV-16 E6 upregulation of DNMT1 through repression of tumor suppressor p53
}

\author{
CHI LAM AU YEUNG, WING PUI TSANG, TSUN YEE TSANG, NGAI NA CO, \\ PAK LUN YAU and TIM TAK KWOK \\ School of Biomedical Sciences, Faculty of Medicine, The Chinese University of Hong Kong, \\ Shatin, Hong Kong SAR, P.R. China
}

Received April 6, 2010; Accepted June 23, 2010

DOI: 10.3892/or_00001023

\begin{abstract}
The HPV-16 early proteins E6 and E7 are considered to function as oncoproteins in cervical cancer. DNA methyltransferase 1 (DNMT1) is one of the enzymes involved in epigenetic silencing of tumor suppressor genes. In the present study, the functional role and regulation of DNMT1 in HPV-16 E6 associated cervical cancer development were examined. Knockdown of E6 in HPV-16 positive human cervical carcinoma $\mathrm{SiHa}$ and CaSki cells led to the increase in $\mathrm{p} 53$, repression of DNMT1 protein and promoter activity. Moreover, p53 knockdown increased the DNMT1 protein as well as promoter activity, indicating that p53 may mediate E6 upregulation of DNMT1. In addition, E6 knockdown induced growth retardation in SiHa cells, and the effect was partially reverted by DNMT1 overexpression. The results suggest that HPV-16 E6 may act through p53/ DNMT1 to regulate the development of cervical cancer.
\end{abstract}

\section{Introduction}

Cervical cancer is the malignant cancer of the cervix or cervical area. It is the second major type of cancer for women worldwide. Cervical cancer is considered as a kind of viral cancer because persistent infection with human papillomavirus (HPV) is regarded as its most important risk factor. Although the infection of HPV does not necessarily cause cervical cancer, it is the prerequisite for its development (1-3).

HPV is a small non-enveloped virus with a doublestranded circular DNA genome of about 8000 base pairs. The virus most likely infects epithelial cells by integration of the viral genome with the host genome $(3,5)$. There are more than 100 genotypes of HPV identified so far. About 40

Correspondence to: Dr Tim Tak Kwok, School of Biomedical Sciences, Faculty of Medicine, The Chinese University of Hong Kong, Room 177, Science Centre, Shatin, Hong Kong SAR, P.R. China

E-mail: kwok2020@cuhk.edu.hk

Key words: HPV-16 E6, DNMT1, p53, cervical cancer of them can be transmitted through sexual contact, infect the genital region, and therefore are categorized as genital HPVs (6). They can be further classified into low-risk or high-risk subtypes based on their oncogenic ability, the degree of risk to develop cancer after infection. Infection with low-risk subtypes of HPVs may develop genital warts while infection with the high-risk subtypes generally may lead to cervical cancer $(7,8)$. Among the high-risk subtypes, HPV-16 causes over $50 \%$ of the cervical cancer cases (2). In terms of cancer development, HPV-16 early proteins E6 and E7 are often believed to act as oncoproteins since both of the proteins are crucial for host cell immortalization and transformation (9). Particularly, E6 targets p53 tumor suppressor directly and enhances p53 degradation via the action of ubiquitin $(1,9,10)$. The process of epigenetic silencing involves the methylation of specific $\mathrm{CpG}$ dinucleotides in the promoter region of the target gene. DNA methyltransferase 1 (DNMT1) is an enzyme that regulates the genomic methylation pattern in cells. Inactivation of tumor suppressor genes (TSGs) through promoter hypermethylation is one of the common events that leads to cancer development and progression (11-14). Meanwhile, overexpression of DNMT1 was often found in human cancers (14-18).

In the present study, the link between HPV-16 E6, p53 and DNMT1 was demonstrated in human cervical carcinoma cells. The findings may provide evidences that DNMT1 is probably involved in HPV-16 mediated human cervical cancer development.

\section{Materials and methods}

Cell lines and reagents. Human cervical carcinoma SiHa cells and CaSki cells were cultured in Dulbecco's modified Eagle's medium (DMEM) supplemented with $10 \%$ fetal bovine serum and $2 \mathrm{mmol} / \mathrm{l} \mathrm{L}$-glutamine (Invitrogen). All cells were incubated in humidified incubator with $10 \% \mathrm{CO}_{2}$ at $37^{\circ} \mathrm{C}$.

Construction of HPV-16 E6 siRNA and full-length HPV-16 E6 cDNA expression vectors. The sequences of the siRNA template oligonucleotides targeting HPV-16 E6 mRNA (from 107 to 127 nucleotides in the gene sequence) were as follows, 5'-AGCTTTTCCAAAAAATGTGTGTACTGCAAGCAA CTCTCTTGAAGTTGCTTGCAGTACACACACGG-3' 


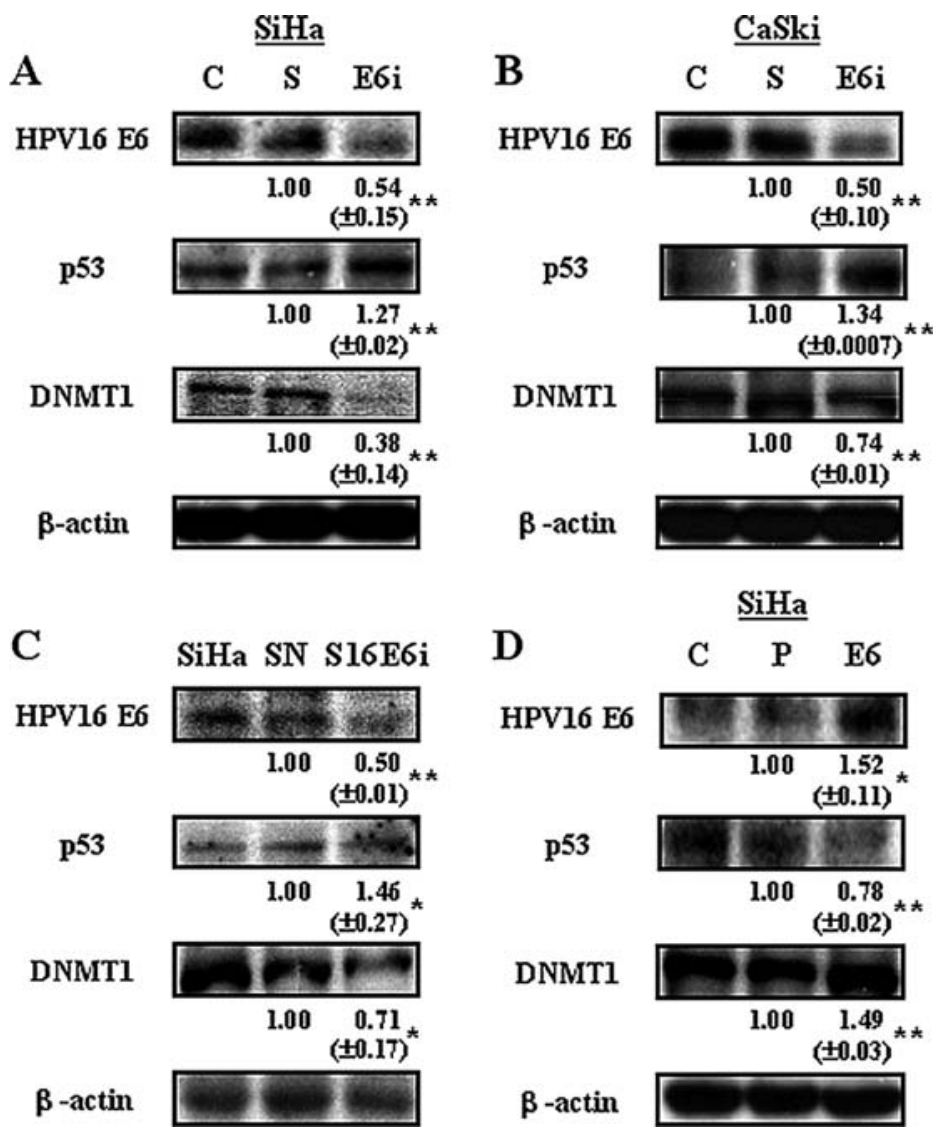

Figure 1. The effect of HPV-16 E6 on p53 and DNMT1 protein expression levels in SiHa and CaSki cells. (A) SiHa cells were transiently transfected with pSilencer empty vector (S) or pSilencer/HPV16E6siRNA (E6i) for $24 \mathrm{~h}$. (B) CaSki cells were transiently transfected with pSilencer empty vector (S) or pSilencer/HPV16E6siRNA (E6i) for 24 h. (C) HPV-16 E6 siRNA stable transfectants. SiHa, SiHa cells without transfection; SN, SiHa cells stably transfected with pSilencer empty vector; S16E6i, SiHa cells stably transfected with pSilencer/HPV16E6siRNA. (D) SiHa cells were transiently transfected with pcDNA3 empty vector (P) or pcDNA3/HPV16E6cDNA (E6) for $24 \mathrm{~h}$. Western blot analysis was performed after protein extraction to determine the protein expression levels. $\beta$-actin was served as the loading control. The relative expression level of each protein was first normalized with that of $\beta$-actin. Results were average from at least three separated experiments. Mean $\pm \mathrm{SD} .{ }^{*} \mathrm{p}<0.05,{ }^{* *} \mathrm{p}<0.01$, compared with the cells with empty vector transfection or SN cells. C, cells with no transfection.

(sense) and 5'-GATCCCGTGTGTGTACTGCAAGCAA CTTCAAGAGAGTTGCTTGCAGTACACACATTTTTTG GAAA-3' (antisense) (1). The annealed hairpin siRNAencoding oligonucleotides were ligated into HindIII and BamHI sites of the pSilencer 2.1-U6 neo vector (Ambion) and sequenced.

The full-length HPV-16 E6 cDNA was amplified by PCR. (Forward primer 5'-GCGGATCCATGCACCAAAA GAGAACTGC-3' and reverse primer 5'-TGCTCGAGTTAC AGCTGGGTTTCTCTAC-3') and then subcloned into the pcDNA3 vector (Invitrogen).

Transfection. All plasmids and p53 siRNA duplex with the sense sequence of 5'-GACUCCAGUGGUAAUCUAC-3' (4) were transfected into cells with the aid of Lipofectamine 2000 (Invitrogen) for $24 \mathrm{~h}$ before sample extraction or MTT cell viability assay.

Establishment of stable transfectants. The plasmid pSilencer/ HPV16E6siRNA and empty pSilencer vector were transfected into SiHa cells using Lipofectamine 2000. Neomycinresistant clones were selected in the presence of $600 \mu \mathrm{g} / \mathrm{ml}$ G418 (Calbiochem). The positive clones were identified by quantitative real-time RT-PCR and Western blot analyses. $\mathrm{SiHa}$ cells stably transfected with pSilencer empty vector were designated as SN cells whereas SiHa cells stably transfected with pSilencer/HPV16E6siRNA with the least expression of HPV-16 E6 was designated as S16E6i cells. Both cells were cultured in complete DMEM medium supplemented with $200 \mu \mathrm{g} / \mathrm{ml} \mathrm{G} 418$.

Western blot analysis. Cells were lysed in lysis buffer containing $1 \%$ Triton X-100, $4.9 \mathrm{mmol} / \mathrm{l} \mathrm{MgCl}_{2}, 1 \mathrm{mmol} / \mathrm{l}$ sodium orthovanadate, $2.1 \mu \mathrm{g} / \mathrm{ml}$ aprotinin, $0.5 \mu \mathrm{g} / \mathrm{ml}$ leupeptin and $1 \mathrm{mmol} / \mathrm{ml}$ phenylmethylsulfonyl fluoride (PMSF). Protein was then resolved by SDS-PAGE according to size and transferred to polyvinylidene fluoride (PVDF) membrane (Millipore) by electroblotting. After overnight blocking with $5 \%$ non-fat dry milk in PBS at $4^{\circ} \mathrm{C}$, the membrane was probed with primary antibodies for $2 \mathrm{~h}$ followed by the respective horseradish peroxidase-conjugated secondary antibodies at 1:10000 dilution. Signals were developed and visualized by using ECL Western blotting reagents (Amersham Biosciences). The protein band intensity was analyzed by ImageJ software (version 1.41o). Primary antibodies against HPV16 E6 (N-17) (Santa Cruz Biotechnology), 
DNMT1 (Epigentek Group), p53 (Merck Biosciences) and $\beta$-actin (Sigma) were used.

Luciferase reporter assay. The part of DNMT1 promoter region containing the p53 consensus binding sequence (at position +30 to +56 relative to the transcription start site) (19) was amplified using the forward primer 5'-TAGGTAC CATCCCCATCACACCTGAAAG-3' and the reverse primer 5'-TAAGAGCTCCCTGTGCAGAAGGATGGAAC-3' and then subcloned into the pGL3 basic vector (Promega) upstream of the luciferase gene. The construct was designated as pGL3/DNMT1pro-p53. The mutated construct pGL3/ DNMT1pro-mutp53 was synthesized using the site-directed mutagenesis kit (Stratagene) with point mutations in the p53

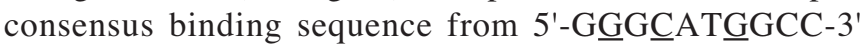
to 5'-GTGTATTGCC-3'. The cells were seeded in a 24-well plate for $24 \mathrm{~h}$ and then transfected with the reporter constructs together with p53 siRNA, pSilencer/HPV16E6siRNA or pcDNA3/HPV16E6cDNA for $24 \mathrm{~h}$. At the same time, control vector containing the Renilla luciferase pRL-CMV was also included in the transfection for monitoring the transfection efficiency. The firefly luciferase activity was measured using the dual-luciferase reporter assay system according to the manufacturer's protocol (Promega). Relative luciferase activity was first normalized with Renilla luciferase activity and then compared with those with the respective control.

MTT cell viability assay. Cells were seeded in a 96-well plate and were allowed to grow for $72 \mathrm{~h}$ after transfection. The cells were then incubated with $50 \mu 1$ of $0.1 \mathrm{mg} / \mathrm{ml} \mathrm{3-(4,5-}$ Dimenthylthiazol-2-yl)-2,5-diphenyltetrazolium bromide (MTT; USB) in PBS at $37^{\circ} \mathrm{C}$ for $3 \mathrm{~h}$. The formazan formed was solubilized with $150 \mu 1$ dimethyl sulfoxide (DMSO). The absorbance was measured at $570 \mathrm{~nm}$ using a microplate reader.

\section{Results}

Effect of HPV-16 E6 on DNMT1 expression. To investigate the effect of the oncoprotein HPV-16 E6 on the expression of DNMT1 in cervical cancer, the DNMT1 protein level in HPV-16 E6 positive SiHa and CaSki cells transiently transfected with HPV-16 E6 siRNA and in SiHa HPV-16 E6 siRNA stable transfectant was examined by Western Blot analysis. Reduced DNMT1 protein expression was observed by knocking down HPV-16 E6 in SiHa and CaSki cells (Fig. 1A and B), as well as in S16E6i cells (Fig. 1C). In contrast, DNMT1 protein level was elevated in HPV-16 E6 overexpressed SiHa cells (Fig. 1D).

Effect of HPV-16 E6 on p53 expression. By knocking down HPV-16 E6 transiently in HPV-16 positive human cervical carcinoma $\mathrm{SiHa}$ and CaSki cells, accumulation of p53 protein was observed (Fig. 1A and B). Similar results were also found in SiHa HPV-16 E6 siRNA stable transfectant, S16E6i cells (Fig. 1C). On the other hand, by full-length HPV-16 E6 cDNA transfection in SiHa cells, a decrease in p53 protein level was resulted (Fig. 1D). The findings for the effect of HPV-16 E6 on p53 were comparable with those reported previously $(1,8)$.
A
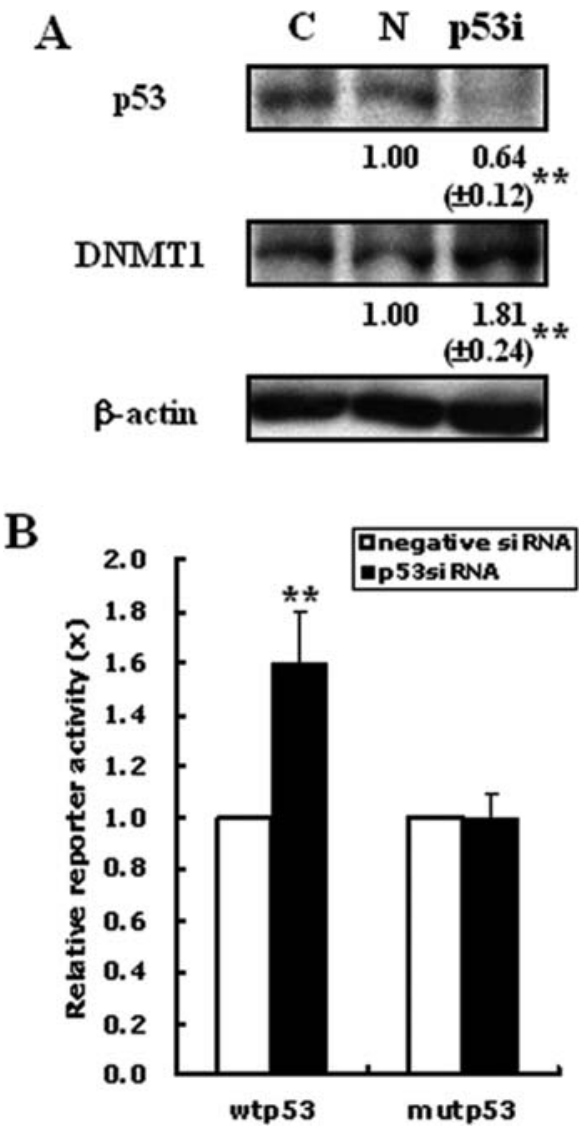

Figure 2. Validation of p53-mediated DNMT1 repression. (A) The effect of p53 siRNA transient transfection on DNMT1 protein expression level in SiHa cells. The cells were transiently transfected with negative siRNA (N) or p53 siRNA (p53i) for $24 \mathrm{~h}$ followed by protein extraction. Western blot analysis was performed to determine the protein expression levels. B-actin served as the loading control. The relative expression level of each protein was first normalized with that of $\beta$-actin. Results were average from at least three separated experiments. Mean \pm SD. ${ }^{* *} \mathrm{p}<0.01$, compared with the cells with negative siRNA transfection. C, cells with no transfection. (B) the effect of p53 siRNA transient transfection on the activity of DNMT1 promoter reporter in SiHa cells. The cells were transiently transfected with pGL3/DNMT1pro-p53 (wtp53) or pGL3/DNMT1pro-mutp53 (mutp53) constructs and negative siRNA or p53 siRNA for $24 \mathrm{~h}$ followed by dualluciferase reporter assay. Results were average from at least three separate experiments. Mean $\pm \mathrm{SD}$. ${ }^{* *} \mathrm{p}<0.01$

Effect of p53 on DNMT1 expression. Knockdown of p53 by p53 siRNA led to an upregulation of DNMT1 in SiHa cells (Fig. 2A). Also, by co-transfecting p53 siRNA with the reporter carrying the DNMT1 promoter with consensus binding sequence of $\mathrm{p} 53$ in $\mathrm{SiHa}$ cells, the reporter activity increased with wild-type p53 binding site, but remained unchanged for that with mutated p53 binding site (Fig. 2B). This shows that p53 may repress the expression of DNMT1.

p53 mediates the HPV-16 E6 upregulation of DNMT1. HPV16 E6 siRNA or full-length HPV-16 E6 cDNA was transfected into SiHa cells together with pGL3 reporter constructs carrying the DNMT1 promoter with either wild-type or mutated p53 binding sites. Knockdown of HPV-16 E6 decreased significantly whereas overexpression of HPV16 E6 increased the reporter activity of DNMT1 promoter containing the wild-type p53 binding site. However, in both cases, there was no change when the DNMT1 promoter 


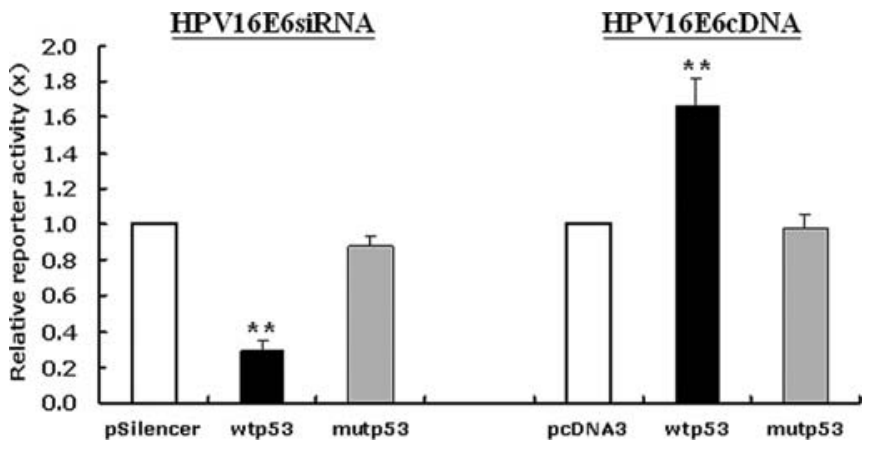

Figure 3. HPV-16 E6 suppresses the p53-mediated repression of DNMT1 in $\mathrm{SiHa}$ cells. The cells were transiently transfected with pGL3/DNMT1prop53 (wtp53) or pGL3/DNMT1pro-mutp53 (mutp53) constructs and pSilencer/ HPV16E6siRNA or pcDNA3/HPV16E6cDNA for $24 \mathrm{~h}$ followed by dualluciferase reporter assay. The cells were transfected with pSilencer or pcDNA3 empty vector in parallel. Results were average from at least three separate experiments. Mean $\pm \mathrm{SD} .{ }^{* *} \mathrm{p}<0.01$.

contained the mutated p53 binding site (Fig. 3). This suggests that HPV-16 E6 may upregulate the expression of DNMT1 through $\mathrm{p} 53$.

DNMT1 suppresses the HPV-16 E6 effect on cell growth. Knockdown of HPV-16 E6 by transient siRNA transfection resulted in the retardation of the growth of $\mathrm{SiHa}$ and CaSki cells down to around 67 and $73 \%$ of the control, respectively (Fig. 4A and B). Comparable results were also found in
SiHa HPV-16 E6 siRNA stable transfectant, S16E6i cells, the growth decreased to $68 \%$ of the control (Fig. 4C). Moreover, increase in cell growth for up to about $130 \%$ of the control was observed in HPV-16 E6 overexpressed SiHa cells (Fig. 4A). The findings were coherent with that reported previously for HPV-16 E6 effect on cell growth $(1,8)$. In addition, the cell growth was increased from 67 to $88 \%$ of the control after the transfection of SiHa cells with HPV-16 E6 siRNA alone and together with DNMT1 cDNA (Fig. 4D), indicating that DNMT1 may mediate the HPV-16 E6 effect on cell growth.

\section{Discussion}

DNA methylation plays a considerable role in cancer progression. Oncogenic viruses, including hepatitis B virus (HBV) $(20,21)$, hepatitis C virus (HCV) (22), Epstein-Barr virus (EBV) $(23,24)$, human T-lymphocyte virus-1 (HTLV-1) (23) and human papillomavirus (HPV) $(14,23,25)$ are often thought to alter the methylation status of cancer cells. In association with cervical carcinogenesis, aberrant promoter methylation and thus the silencing of various tumor suppressor genes (TSGs) was reported, such as p16INK4a (25-27), RASSF1A (27), E-cadherin $(25,28)$ and deathassociated protein kinase (DAPK) (25). DNA methyltransferase 1 (DNMT1) was believed to be the major DNA methyltransferase involved in DNA methylation (14). Increased DNMT1 activity and expression were found in human cancers $(15,16)$, including cervical cancer $(14,18,29)$.
A

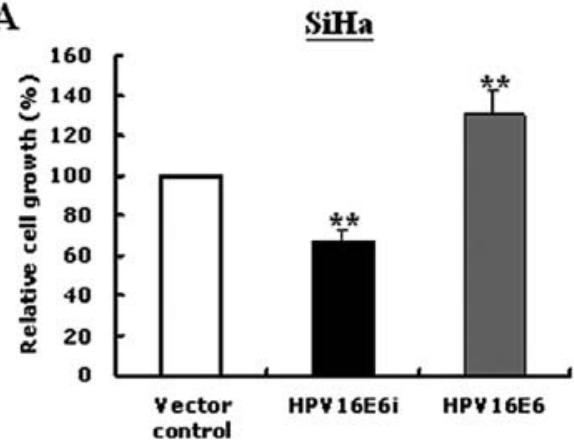

B

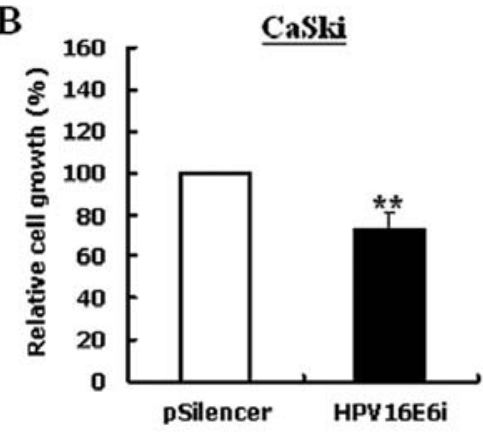

C

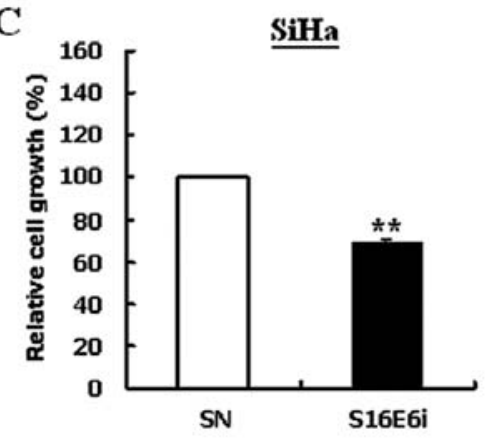

D

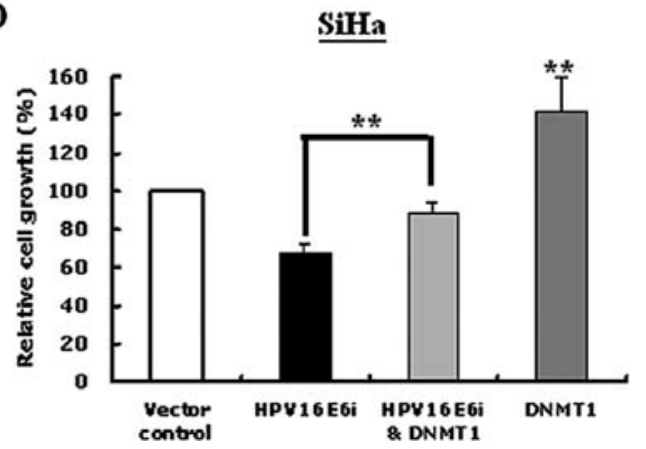

Figure 4. The effect of HPV-16 E6 and DNMT1 on the growth of SiHa and CaSki cells. (A) SiHa cells were transiently transfected with pSilencer/ HPV16E6siRNA (HPV16E6i) or pcDNA3/HPV16E6cDNA (HPV16E6). The cells were transfected with their corresponding empty vector in parallel. (B) CaSki cells were transiently transfected with pSilencer empty vector (pSilencer) or pSilencer/HPV16E6siRNA (HPV16E6i). (C) HPV-16 E6 siRNA stable transfectants, SN and S16E6i cells. (D) SiHa cells were transiently transfected with pSilencer/HPV16E6siRNA and/or pGFP-C3/DNMT1cDNA (HPV16E6i and DNMT1, HPV16E6i or DNMT1). The cells were allowed to grow for $72 \mathrm{~h}$ after transfection. MTT assay was performed to determine the cell viability. Results were average from at least three separate experiments. Mean $\pm \mathrm{SD} .{ }^{* *} \mathrm{p}<0.01$. 
The increase in DNMT1 will likely be induced by the oncoprotein HPV-16 E6 in human cervical cancer cells (Fig. 1).

The tumor suppressor p53 is one of the most wellcharacterized target genes of HPV-16 E6 $(8,30,31)$ besides the PDZ domain containing proteins (25), human telomerase reverse transcriptase (hTERT) (32), vascular endothelial growth factor (VEGF) $(33,34)$, and hypoxia-inducible factor $1 \alpha(\mathrm{HIF}-1 \alpha)$ (34). p53 was recently reported to repress the DNMT1 level in human colon carcinoma HCT116 cells (19). Similarly, knockdown of p53 also upregulated DNMT1 in SiHa cells (Fig. 2A). The regulatory role of p53 in DNMT1 is further confirmed as co-transfection with p53 siRNA in SiHa cells increased the activity of the DNMT1 promoter reporter with wild-type p53 binding site, while has no effect on that with mutated p53 binding site (Fig. 2B).

HPV-16 E6 was found to upregulate DNMT1 and suppress p53 in cells (Fig. 1). On the other hand, p53 also represses the DNMT1 level (Fig. 2). It is therefore hypothesized that HPV-16 E6 may act through p53 to induce DNMT1 level in HPV-16 positive human cervical carcinoma cells. To verify this hypothesis, the activity of DNMT1 promoter reporter transfected together with either HPV-16 E6 siRNA or full-length HPV-16 E6 cDNA was examined. A significant decrease in the activity of reporter with wild-type p53 binding site was observed in HPV-16 E6 knockdown $\mathrm{SiHa}$ cells, though an increase was found for HPV-16 E6 overexpression. There was no change in the activity for the DNMT1 promoter with mutated p53 binding site (Fig. 3). This suggests that p53 may mediate HPV-16 E6 upregulation of DNMT1.

One of the major biological functions of HPV-16 E6 in cervical cancer development is induction of cell growth (Fig. 4A-C). As DNMT1 was found to be upregulated by HPV-16 E6, the significance of the pathway in cell growth was therefore examined by co-transfection of SiHa cells with HPV-16 E6 siRNA and DNMT1 cDNA. As shown in Fig. 4D, DNMT1 may counteract the growth suppression effect induced by HPV-16 E6 knockdown. The results therefore support that DNMT1 may be one of the downstream molecules in mediating the effect of HPV-16 E6 on cell growth.

In conclusion, oncogenic E6 protein of high-risk HPV-16 regulates the expression of DNMT1 through tumor suppressor p53. Although the present study only indicates the possible role of the DNMT1 in E6 regulation of cell growth, further investigation is necessary to clarify the significance of the pathway between HPV-16 E6 and DNMT1 in cervical cancer development. The information from such study may help to provide new perspective on developing new strategies for curing cervical cancer.

\section{Acknowledgements}

The study is supported by grants from Hong Kong Research Grants Council General Research Fund 466908, 467609.

\section{References}

1. Bai L, Wei L, Wang J, Li X and He P: Extended effects of human papillomavirus 16 E6-specific short hairpin RNA on cervical carcinoma cells. Int J Gynecol Cancer 16: 718-729, 2006.
2. Walboomers JM, Jacobs MV, Manos MM, et al: Human papillomavirus is a necessary cause of invasive cervical cancer worldwide. J Pathol 189: 12-19, 1999.

3. Munger K, Baldwin A, Edwards KM, et al: Mechanisms of human papillomavirus-induced oncogenesis. J Virol 78: 11451-11460, 2004.

4. Brummelkamp TR, Bernards R and Agami R: A system for stable expression of short interfering RNAs in mammalian cells. Science 296: 550-553, 2002.

5. zur Hausen H: Papillomaviruses and cancer: from basic studies to clinical application. Nat Rev Cancer 2: 342-350, 2002.

6. Soliman PT, Slomovitz BM and Wolf JK: Mechanisms of cervical cancer. Drug Discovery Today 1: 253-258, 2004.

7. Munoz N, Bosch FX, de Sanjose S, et al: Epidemiologic classification of human papillomavirus types associated with cervical cancer. N Engl J Med 348: 518-527, 2003.

8. Zuna RE, Allen RA, Moore WE, Mattu R and Dunn ST: Comparison of human papillomavirus genotypes in high-grade squamous intraepithelial lesions and invasive cervical carcinoma: evidence for differences in biologic potential of precursor lesions. Mod Pathol 17: 1314-1322, 2004

9. Jiang $M$ and Milner J: Selective silencing of viral gene expression in HPV-positive human cervical carcinoma cells treated with siRNA, a primer of RNA interference. Oncogene 21: 6041-6048, 2002 .

10.Tang S, Tao M, McCoy JP Jr and Zheng ZM: Shortterm induction and long-term suppression of HPV16 oncogene silencing by RNA interference in cervical cancer cells. Oncogene 25: 2094-2104, 2006.

11. Kanai Y and Hirohashi S: Alterations of DNA methylation associated with abnormalities of DNA methyltransferases in human cancers during transition from a precancerous to a malignant state. Carcinogenesis 28: 2434-2442, 2007.

12. Paz MF, Wei S, Cigudosa JC, et al: Genetic unmasking of epigenetically silenced tumor suppressor genes in colon cancer cells deficient in DNA methyltransferases. Hum Mol Genet 12: 2209-2219, 2003.

13. Suzuki M, Sunaga N, Shames DS, Toyooka S, Gazdar AF and Minna JD: RNA interference-mediated knockdown of DNA methyltransferase 1 leads to promoter demethylation and gene re-expression in human lung and breast cancer cells. Cancer Res 64: 3137-3143, 2004.

14. Jacinto FV, Ballestar E, Ropero S and Esteller M: Discovery of epigenetically silenced genes by methylated DNA immunoprecipitation in colon cancer cells. Cancer Res 67: 11481-11486, 2007.

15. Sawada M, Kanai Y, Arai E, Ushijima S, Ojima H and Hirohashi S: Increased expression of DNA methyltransferase 1 (DNMT1) protein in uterine cervix squamous cell carcinoma and its precursor lesion. Cancer Lett 251: 211-219, 2007.

16. Issa JP, Vertino PM, Wu J, et al: Increased cytosine DNAmethyltransferase activity during colon cancer progression. J Natl Cancer Inst 85: 1235-1240, 1993.

17. Sun L, Hui AM, Kanai Y, Sakamoto M and Hirohashi S: Increased DNA methyltransferase expression is associated with an early stage of human hepatocarcinogenesis. Jpn J Cancer Res 88: 1165-1170, 1997.

18. Hino R, Uozaki H, Murakami N, et al: Activation of DNA methyltransferase 1 by EBV latent membrane protein $2 \mathrm{~A}$ leads to promoter hypermethylation of PTEN gene in gastric carcinoma. Cancer Res 69: 2766-2774, 2009.

19. Peterson EJ, Bogler O and Taylor SM: p53-mediated repression of DNA methyltransferase 1 expression by specific DNA binding. Cancer Res 63: 6579-6582, 2003.

20. Su PF, Lee TC, Lin PJ, et al: Differential DNA methylation associated with hepatitis B virus infection in hepatocellular carcinoma. Int J Cancer 121: 1257-1264, 2007.

21. Chan KC, Lai PB, Mok TS, et al: Quantitative analysis of circulating methylated DNA as a biomarker for hepatocellular carcinoma. Clin Chem 54: 1528-1536, 2008.

22. Nishida N, Nagasaka T, Nishimura T, Ikai I, Boland CR and Goel A: Aberrant methylation of multiple tumor suppressor genes in aging liver, chronic hepatitis, and hepatocellular carcinoma. Hepatology 47: 908-918, 2008.

23. Burgers WA, Blanchon L, Pradhan S, de Launoit Y, Kouzarides T and Fuks F: Viral oncoproteins target the DNA methyltransferases. Oncogene 26: 1650-1655, 2007.

24. Uozaki H and Fukayama M: Epstein-Barr virus and gastric carcinoma-viral carcinogenesis through epigenetic mechanisms. Int J Clin Exp Pathol 1: 198-216, 2008. 
25. Dong SM, Kim HS, Rha SH and Sidransky D: Promoter hypermethylation of multiple genes in carcinoma of the uterine cervix. Clin Cancer Res 7: 1982-1986, 2001.

26. Virmani AK, Muller C, Rathi A, Zoechbauer-Mueller S, Mathis $\mathrm{M}$ and Gazdar AF: Aberrant methylation during cervical carcinogenesis. Clin Cancer Res 7: 584-589, 2001.

27. Narisawa-Saito M and Kiyono T: Basic mechanisms of highrisk human papillomavirus-induced carcinogenesis: roles of E6 and E7 proteins. Cancer Sci 98: 1505-1511, 2007.

28. Chen CL, Liu SS, Ip SM, Wong LC, Ng TY and Ngan HY: Ecadherin expression is silenced by DNA methylation in cervical cancer cell lines and tumours. Eur J Cancer 39: 517-523, 2003.

29. Woodman CB, Collins SI and Young LS: The natural history of cervical HPV infection: unresolved issues. Nat Rev Cancer 7: $11-22,2007$

30. Scheffner M, Werness BA, Huibregtse JM, Levine AJ and Howley PM: The E6 oncoprotein encoded by human papillomavirus types 16 and 18 promotes the degradation of $\mathrm{p} 53$. Cell 63: 1129-1136, 1990
31. Scheffner M, Huibregtse JM, Vierstra RD and Howley PM: The HPV-16 E6 and E6-AP complex functions as a ubiquitinprotein ligase in the ubiquitination of p53. Cell 75: 495-505, 1993.

32. Veldman T, Horikawa I, Barrett JC and Schlegel R: Transcriptional activation of the telomerase hTERT gene by human papillomavirus type 16 E6 oncoprotein. J Virol 75: 4467-4472, 2001

33. Lopez-Ocejo O, Viloria-Petit A, Bequet-Romero M Mukhopadhyay D, Rak J and Kerbel RS: Oncogenes and tumor angiogenesis: the HPV-16 E6 oncoprotein activates the vascular endothelial growth factor (VEGF) gene promoter in a p53 independent manner. Oncogene 19: 4611-4620, 2000.

34. Tang X, Zhang Q, Nishitani J, Brown J, Shi S and Le AD: Overexpression of human papillomavirus type 16 oncoproteins enhances hypoxia-inducible factor 1 alpha protein accumulation and vascular endothelial growth factor expression in human cervical carcinoma cells. Clin Cancer Res 13: 2568-2576, 2007. 